\title{
Exploitation of Contrasts in Low Energy SEM to Reveal True Microstructure
}

\author{
Ilona Müllerová, Šárka Mikmeková, Eliška Mikmeková, Zuzana Pokorná and Luděk Frank \\ Department of Electron Microscopy, Institute of Scientific Instruments ASCR v.v.i., Brno, Czech \\ Republic.
}

We have developed a Scanning Low Energy Electron Microscope (SLEEM) based on the Cathode Lens (CL) principle [1]. A resolution of $4.5 \mathrm{~nm}$ at $20 \mathrm{eV}, 0.8 \mathrm{~nm}$ at $200 \mathrm{eV}$ and $0.5 \mathrm{~nm}$ at $15 \mathrm{keV}$ primary beam energy can nowadays be obtained in a commercially available instrument [2]. One of the main advantages of operation at low energies is the decrease in the interaction volume from approximately 1 $\mu \imath \eta$ at $10 \mathrm{keV}$ to $10 \mathrm{~nm}$ at $100 \mathrm{eV}$. The material contrast can be optimised and the charging effect suppressed at a tailored electron energy. Wave-optical contrasts are also available beneath $50 \mathrm{eV}$. The specimen may be immersed in a strong magnetic field in addition to an electrostatic field in order to obtain a small spot size across the whole energy range. The same fields influence the signal trajectories, so we can choose which part of the angular and energy distributions of emitted electrons are to be collected. Certain arrangements provide strong crystallographic contrast. Imaging conditions have been tailored to various material types. Experiments have been performed in an experimental ultrahigh vacuum (UHV) SLEEM and in an XHR SEM Magellan 400L.

An example of the imaging of chromium ledeburitic steel X210Cr12 in a UHV SLEEM is shown in Fig. 1. The specimen was cleaned in-situ for 60 minutes by $3 \mathrm{keV}$ Ar ions at an incidence angle of $10^{\circ}$ with respect to the surface. Micrographs of this steel show an improvement in the crystallographic contrast with the decreasing landing energy of electrons and demonstrate strain distribution in a heavily deformed specimen. The influence of the fields surrounding the specimen on the final image contrast is demonstrated in Fig. 2. Both pictures are taken at the same energy of $2 \mathrm{keV}$ and by the same detector, but the collection of signal electrons to the detector is significantly improved by the presence of the electrostatic field above the sample surface. When electrons emitted at a large angle from the optical axis are acquired we obtain a higher contrast of precipitates as well as the surface topography, see Fig. 2b. Similarly, strongly enhanced surface sensitivity at very low energies is apparent in Fig. 3 . In this case, the complete emission of backscattered electrons is collected with the exception of the axial ray. The reflection of very slow electrons from crystals is known to be inversely proportional to the local density of free electron states in the direction of motion, which is characteristic of the crystallographic system and its orientation. Thus, when acquiring a data-cube of frames along the energy scale (see Fig. 4), we can identify the orientation of grains in polycrystals. The method was confirmed by means of EBSD, with respect to which we achieve higher resolution and faster data acquisition [3].

When optimising the energy of incident electrons and the energy and angular ranges of detected signal electrons we can significantly enhance the information content of SEM images. [4]

\section{References:}

[1] I Müllerová and L Frank, Advances in Imaging and Electron Physics 128 (2003), p. 309.

[2] I Müllerová et al, Microscopy and Microanalysis 19 S2 (2013), p. 1236.

[3] Z Pokorná et al, Applied Physics Letters 100 (2012), 261602. 
[4] The authors acknowledge funding from the Technology Agency of the Czech Republic (Competence center Electron microscopy, no: TE01020118) and from the MEYS of the Czech Republic (LO1212) together with the European Commission (ALISI, no. CZ.1.05/2.1.00/01.0017).

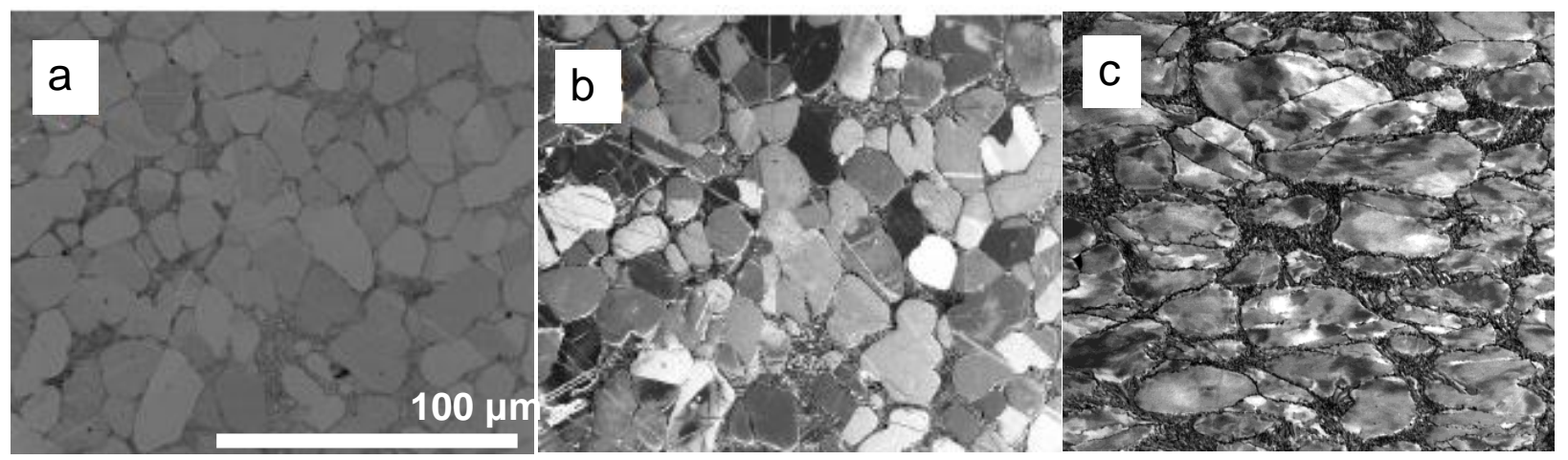

Figure 1. UHV SLEEM images of the X210Cr12 steel obtained at $6018 \mathrm{eV}$ (a) and $500 \mathrm{eV}$ (b), and image of the heavily deformed sample at $500 \mathrm{eV}$ (c).

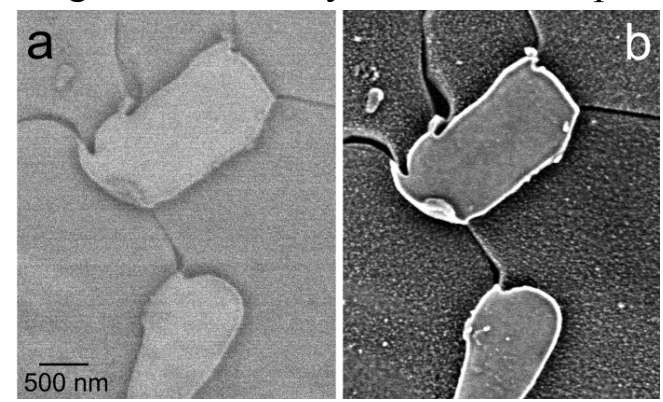

Figure 2. Mg17Al12 precipitates in the AZ91 alloy imaged at $2 \mathrm{keV}$ : (a) Standard imaging with backscattered electrons acquired between polar angles $10^{\circ}$ and $45^{\circ}$; (b) sample immersed in 450 $\mathrm{V} / \mathrm{mm}$ field, polar angles $13^{\circ}$ to $63^{\circ}$ acquired.

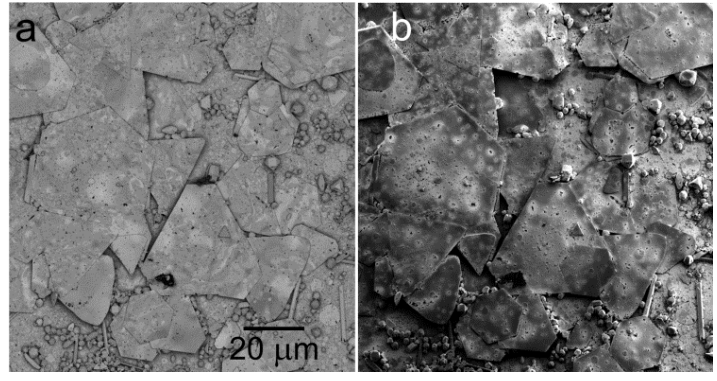

Figure 3. Microcrystalline Au deposited on glass: (a) Standard BSE imaging at $6 \mathrm{keV}$; (b) Sample in $1 \mathrm{kV} / \mathrm{mm}$, landing energy of electrons $5 \mathrm{eV}$.
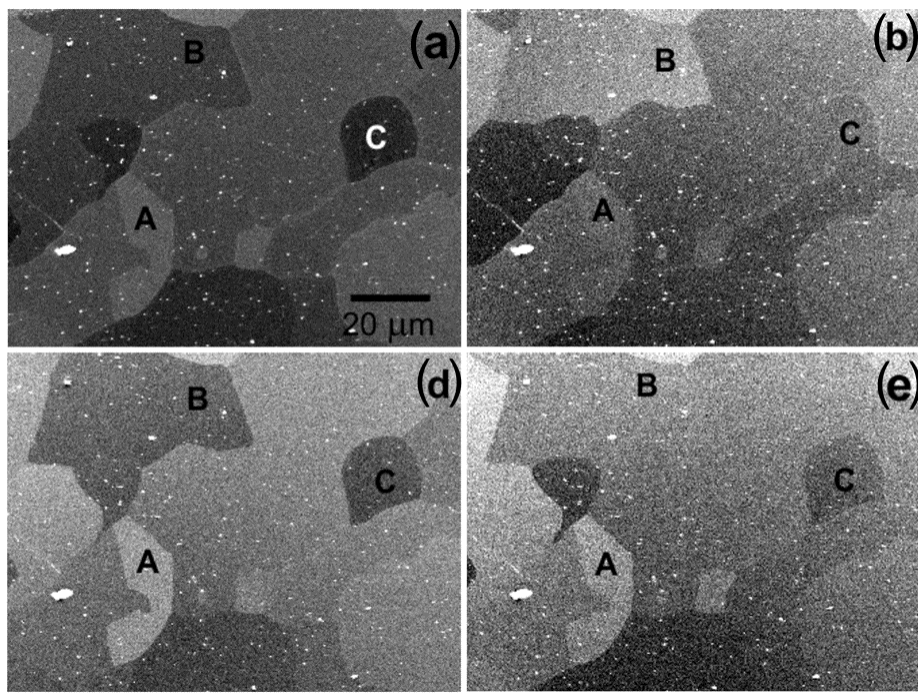

(b)
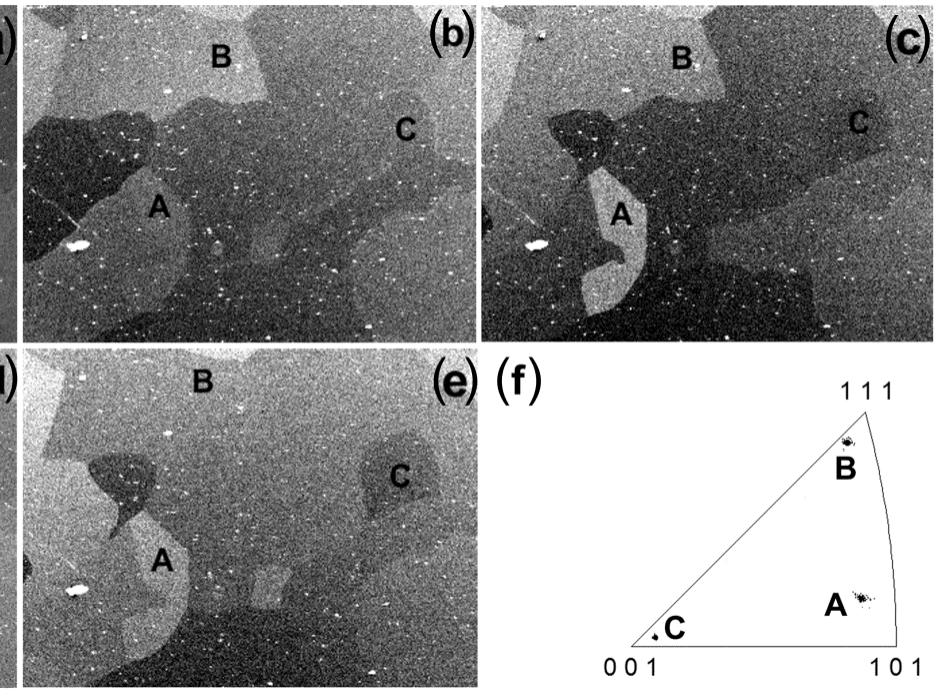

(e) (f)

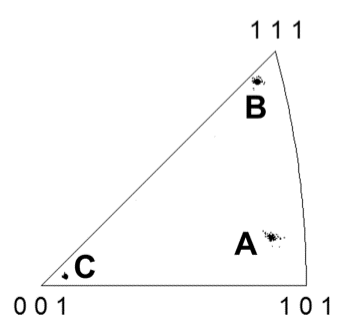

Figure 4. Micrographs of a clean and restored polycrystalline aluminum surface shown at electron energies $7.8 \mathrm{eV}$ (a), $17.4 \mathrm{eV}$ (b), $24.6 \mathrm{eV}$ (c), $30.9 \mathrm{eV}$ (d), and $40.8 \mathrm{eV}$ (e), together with the inverse pole map containing pixels from three grains marked as $\mathrm{A}, \mathrm{B}$, and $\mathrm{C}(\mathrm{f})$. 